\title{
A search for planetary-mass objects and brown dwarfs in the Upper Scorpius association ${ }^{\star}$
}

\author{
M. T. Costado ${ }^{1}$, V. J. S. Béjar ${ }^{2}$, J. A. Caballero ${ }^{1}$, R. Rebolo ${ }^{1,3}$, J. Acosta-Pulido ${ }^{1}$, and A. Manchado ${ }^{1,3}$ \\ ${ }^{1}$ Instituto de Astrofísica de Canarias, 38205 La Laguna, Tenerife, Spain \\ 2 Instituto de Astrofísica de Canarias. Gran Telescopio Canarias Project, Spain \\ e-mail: vbejar@iac.es \\ ${ }^{3}$ Consejo Superior de Investigaciones Científicas, Spain
}

Received 11 March 2005 / Accepted 3 August 2005

\begin{abstract}
We report the results of a deep photometric search for planets and brown dwarfs in the nearby young OB Upper Scorpius association. We obtained optical $(I)$ and near-infrared $\left(J K_{\mathrm{s}}\right)$ images around nine very low-mass stars and brown dwarf member candidates of the association, covering a total area of $113 \mathrm{arcmin}^{2}$. Using a point spread function subtraction technique, we have searched for planetary-mass companions $\left(0.002 M_{\odot}<M<0.013 M_{\odot}\right)$ at separations as close as $3^{\prime \prime}$ from the targets. We have not found any brown dwarfs more massive than $0.030 M_{\odot}$ at projected distances larger than $70 \mathrm{AU}$, or planets more massive than $0.007 M_{\odot}$ at projected distances larger than $600 \mathrm{AU}$. We set an upper limit of $20 \%$ (confidence level $68.3 \%$ ) to the fraction of very low-mass stars and massive brown dwarfs with planetary mass companions $\left(M>0.007 M_{\odot}\right)$ at physical distances larger than $600 \mathrm{AU}$. From the $J, I-J$ colour-magnitude diagrams and follow-up $K_{\mathrm{s}}$-band photometry, we identify four very red objects $(I-J>2.5)$ in the area of the survey. According to their positions in the diagram, below the expected theoretical sequence of the cluster, three are probable late M-dwarfs in the field. The faintest and reddest object $\left(J-K_{\mathrm{s}}=2.39 \pm 0.06\right)$ may be either a field intermediate L-dwarf with anomalously red $J-K_{\mathrm{s}}$ colour at a distance of $\sim 135 \mathrm{pc}$, an extremely red distant galaxy, or a reddened planetary-mass object in the Upper Scorpius association.
\end{abstract}

Key words. stars: low mass, brown dwarfs - open clusters and associations: individual: Upper Scorpius

\section{Introduction}

The Upper Scorpius OB association, located at a distance of $145 \pm 2$ pc (de Zeeuw et al. 1999) and with an estimated age of 5-6 Myr (Preibisch \& Zinnecker 1999), belongs to the Scorpio-Centaurus-Lupus-Crux complex. The galactic latitude is in the range $+19-21^{\circ}$. The characteristics of youth, proximity and low extinction makes Upper Scorpius an ideal site to search for very low-mass stars and substellar objects. Recent studies by Ardila et al. (2000) and Preibisch et al. $(2001,2002)$ have investigated the population of very low-mass stars and brown dwarfs in the Upper Scorpius. Many of them have been confirmed as bona fide members of the association and several show evidence of the presence of accretion discs (Jayawardhana et al. 2002, 2003).

In this paper we present deep images in the optical $I$ and infrared $J K_{\mathrm{s}}$-bands of low-mass stars and brown dwarfs in the Upper Scorpius association with sufficient sensitivity to directly detect planetary-mass companions at wide separations.

* Appendices A-C are only available in electronic form at http://www.edpsciences.org

\section{Sample selection and observations}

We selected nine faint and cool potential members of the Upper Scorpius association from studies by Ardila et al. (2000) and Preibisch et al. (2001). The four objects selected from Preibisch et al. (2001) are very low-mass stars of spectral type M5, with lithium in absorption and $\mathrm{H} \alpha$ in emission, so they are very likely members of this association. Three out of the five objects selected from Ardila et al. (2000) were later confirmed as bona fide members through the detection of lithium in their spectra. They are very low-mass stars or massive brown dwarfs with spectral types later than M 5. They also display $L^{\prime}$-band excess and/or very intense and broad $\mathrm{H} \alpha$ emission lines that suggest the existence of discs. Subsequent studies to our observations have shown that one of the remaining candidates (UScoCTIO 121) has depleted its lithium (Muzerolle et al. 2003) and is probably a background field dwarf star. Names, coordinates, photometric and main spectral properties for these objects are given in Tables A.1 and A.2 (both tables are only available in the electronic version in Appendix A).

We have performed optical $(I)$ and near-infrared $\left(J K_{\mathrm{s}}\right)$ observations. A log of the observations is presented in Table 1. 
Table 1. Log of the observations.

\begin{tabular}{|c|c|c|c|c|c|c|c|}
\hline Filter & Instrument & Telescope & Detector & Scale & Bin & Seeing & Date \\
\hline$I$ & OIG & TNG & $2048 \times 4096 \mathrm{EEV}$ & 0.072 arcsec/pixel & $2 \times 2$ & $0.6-0.9$ & 02 June $28-30$ \\
\hline$J$ & INGRID & WHT & $1024 \times 1024$ pixel $^{2}$ Hawai'i & $0.238 \mathrm{arcsec} / \mathrm{pixel}$ & $1 \times 1$ & 1.1 & 02 March 26 \\
\hline$K_{\mathrm{s}}$ & CAIN-2 & TCS & $256 \times 256$ Nicmos- 3 & 1 arcsec/pixel & $1 \times 1$ & 1.5 & 02 June $7-9$ \\
\hline$K_{\mathrm{s}}$ & LIRIS & WHT & $1024 \times 1024$ pixel $^{2}$ Hawai'i & 0.25 arcsec/pixel & $1 \times 1$ & $1.2-1.5$ & 04 June 8-10 \\
\hline
\end{tabular}

Table 2. Detected visual companions to our targets.

\begin{tabular}{lcccccc}
\hline \hline USco primary & $\begin{array}{c}\rho \\
\left({ }^{\prime}\right)\end{array}$ & $\begin{array}{c}\theta \\
(\mathrm{deg})\end{array}$ & $I_{\mathrm{obj}}$ & $(I-J)_{\mathrm{obj}}$ & $(I-J)_{\text {USco }}$ & $I_{\text {obj }}-I_{\text {USco }}$ \\
& $3.00 \pm 0.05$ & $201.3 \pm 0.6$ & $20.0 \pm 0.4$ & $0.5 \pm 0.4$ & $2.07 \pm 0.05$ & $6.0 \pm 0.4$ \\
\hline UScoCTIO 56 & $4.80 \pm 0.05$ & $339.9 \pm 0.5$ & $20.8 \pm 0.4$ & $1.7 \pm 0.4$ & $1.85 \pm 0.06$ & $7.5 \pm 0.4$ \\
USco J160628.7-200357 & & & \\
USco J160629.0-205216 & $5.10 \pm 0.05$ & $354.4 \pm 0.3$ & $21.3 \pm 0.4$ & $1.8 \pm 0.4$ & $2.20 \pm 0.05$ & $8.0 \pm 0.4$ \\
\hline
\end{tabular}

Standard reduction processes and aperture and psf photometry have been performed. For the calibration of the $J$ and $K_{\mathrm{s}}$-band, we used the photometry from the 2MASS catalogue (Cutri et al. 2003) and for the $I$-band data we used the photometry from the DENIS catalogue (DENIS Consortium 2003, Epchtein et al. 1994). We have transformed the $I$-band data from DENIS into the Cousins system, using $I_{\mathrm{C}}-i_{\text {DENIS }}=0.030 \pm 0.04$, obtained by comparison of the photometry of $\mathrm{M}$ and $\mathrm{L}$ dwarfs from Legget et al. (2000, 2001) and DENIS. Estimates for the completeness and limiting magnitudes (with an error of $0.5 \mathrm{mag}$ ) of the $I-, J-, K_{\mathrm{S}}(\mathrm{TCS})-$ and $K_{\mathrm{S}}$ (LIRIS)-band images are 21.6, 19.2, $17.1,18.5$ and $22.5,20.0,17.8,19.5$ respectively. For more details see the electronic version in Appendix B.

\section{Results and discussion}

\subsection{Search for nearby companions}

We have performed a search for faint companions at close angular distances, $\rho<10$ arcsec, to the selected targets (equivalent to a projected distance $r<1500 \mathrm{AU}$ ). Because of the depth of our images, the primaries are very close to saturation and their wings are very strong and extended. To detect possible faint companions, we have implemented a PSF subtraction method consisting basically of the subtraction to the original image of the rotated image centred on the primary. We applied a rotation of $180^{\circ}$ because this rotation reflects the symmetry of the PSF quite well and determined the centre of rotation by minimizing the resulting residuals of the subtraction of the wings of the central object. We have found only three possible candidates located at separations of 3 to 5 arcsec from three primaries (see Fig. 1 and Table 2). These candidates are detected both in the $I$ and $J$ images, but not in the $K_{\mathrm{s}}$ images because of the lower resolution of the images taken with CAIN-2.

We have performed aperture photometry of these candidates in the PSF subtracted image. The magnitude $I$ and the colour $(I-J)$ of the close visual companions (obj), of their primaries (USco) and the difference in magnitude of both are shown in Table 2. Although uncertainties in the $I J$-band magnitudes of the visual companions are very large, the candidates are bluer than the primary central objects, opposite to what is
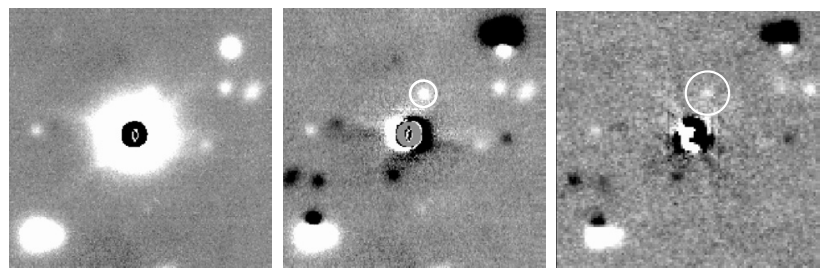

Fig. 1. $I$ - and $J$-band images of USco J160628.7-200357 and its nearby visual companion, marked with a white circle. We show the $I$-band images prior to PSF subtraction in the left window. PSF-subtracted $I$ - and $J$-band images are shown in the central and right windows, respectively. The size of the images are $30 \times 30 \operatorname{arcsec}^{2}$. North up, and east is to left.

expected if they were real companions. Hence they seem to be just field objects.

\subsection{Detectability of nearby companions}

We have estimated the faintest magnitude (i.e. lowest mass) that can be detected at a given distance of a target in our images. We have generated artificial objects with different magnitudes using the mkobjects routine within IRAF. After PSF subtraction, using the method explained in the previous section, we determined the magnitude of the faintest object that can be detected by visual inspection. This analysis was repeated for a range of distances to the central object until a distance where there is no major contribution from the primary wings and the sources are detected at the limiting magnitude of the survey.

We have transformed $J$-band magnitude to masses, using the theoretical isochrone of $5 \mathrm{Myr}$ from the Lyon group (Baraffe et al. 1998; Chabrier et al. 2000), and a distance modulus $J-M_{J}=5.81 \pm 0.03$ for the Upper Scorpius association (de Zeeuw et al. 1999). We have converted the luminosity and $T_{\text {eff }}$ given by the theoretical models into magnitudes using the bolometric corrections and $T_{\text {eff }}$ vs. spectral type from Golimowski et al. (2004), and the spectral type vs. $I-J$ colour relation from Leggett et al. (2000, 2001). In Fig. 2, we show the minimum masses of the companions that can be detected at angular separations between $0.5 \operatorname{arcsec}(\sim 70 \mathrm{AU})$ and $5 \operatorname{arcsec}$ (750 AU). The mass of the central object is included at the null 
Table 3. Ultracool objects.

\begin{tabular}{lccccccc}
\hline \hline $\begin{array}{l}\text { Id. } \\
\text { number }\end{array}$ & $\begin{array}{c}\alpha \\
(\mathrm{J} 2000)\end{array}$ & $\begin{array}{c}\delta \\
(\mathrm{J} 2000)\end{array}$ & $I$ & $I-J$ & $J-K_{\mathrm{s}}$ & $\begin{array}{c}\text { Expected } \\
\text { Sp.T. }{ }^{a}\end{array}$ & Field \\
\hline$\# 1$ & 160626.0 & -201152 & $22.5 \pm 0.5$ & $2.9 \pm 0.5$ & $<2.3$ & M 7 to L 6 & USco J160622.8-201124 \\
$\# 2$ & 160625.2 & -200441 & $22.22 \pm 0.09$ & $3.04 \pm 0.09$ & $<1.9$ & M 8: & USco J160628.7-200357 \\
$\# 3$ & 160631.2 & -205350 & $21.43 \pm 0.08$ & $3.07 \pm 0.09$ & $1.01 \pm 0.09$ & M 8: & USco J160629.0-205216 \\
$\# 4$ & 160629.1 & -201928 & $23.0 \pm 0.5$ & $3.6 \pm 0.5$ & $2.39 \pm 0.1$ & L 5-L 7: & USco J160632.1-202053 \\
\hline
\end{tabular}

${ }^{a}$ Estimated spectral types assuming that the objects are dwarfs.

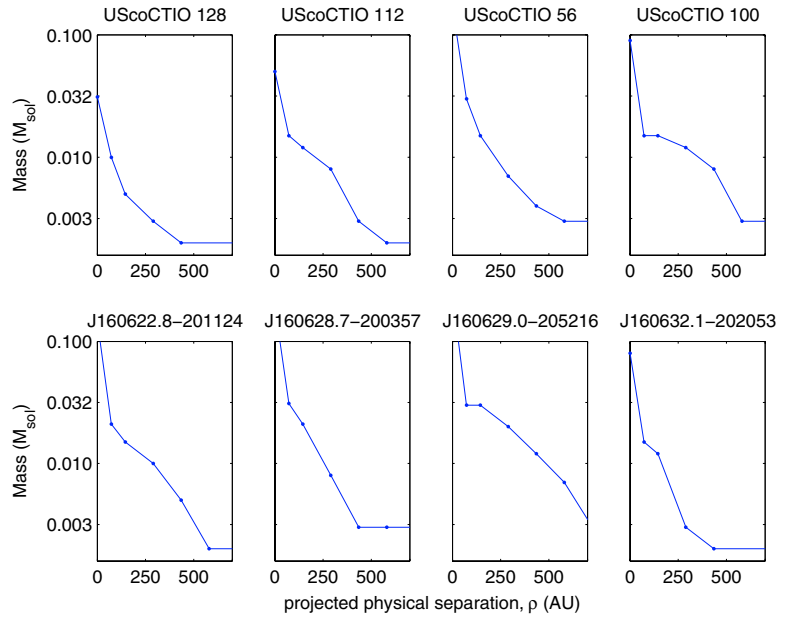

Fig. 2. The minimum mass of detectable companions at a given distance of the targets. The vertical scale is logarithmic.

distance. We have the sensitivity to detect planets more massive than $0.002 M_{\odot}\left(\sim 2 M_{\text {Jup }}\right)$ and brown dwarfs at angular separations larger than 3-4 arcsec (450-600 AU) and 0.5-3 arcsec ( $70-450 \mathrm{AU})$, respectively. In conclusion, we have not found any brown dwarfs more massive than $0.030 M_{\odot}$ at projected distances larger than $70 \mathrm{AU}$, or any planets more massive than $0.007 M_{\odot}$ at projected distances larger than $600 \mathrm{AU}$.

As we have not found any physical companion, we can only determine an upper limit of $14.3 \%$ (for a confident level of $68.3 \%$ ) to the real fraction of substellar companions at the distances and masses mentioned above (see the Appendix $\mathrm{C}$ in electronic version for more details). In order to know the real fraction of substellar companions at physical (not projected) distances, it is necessary to correct this result by the probability that the orbital position of the companion is at a detectable projected distance. Taking into account this correction, we can determine an upper limit of $20 \%$ on the fraction of very lowmass stars and brown dwarfs with planetary companions $(M>$ $0.007 M_{\odot}$ ) at physical distances larger than $\sim 600 \mathrm{AU}$ and with brown dwarf companions at distances larger than $\sim 400 \mathrm{AU}$. Less restrictive upper limits can be set for shorter distances. These results together with McCarthy \& Zuckerman (2004) seem to indicate that substellar objects are rare companions of low-mass stars. The discovery of a planet candidate by Chauvin et al. (2004), however, shows that the probability of finding planets around substellar objects could be low, but not null.

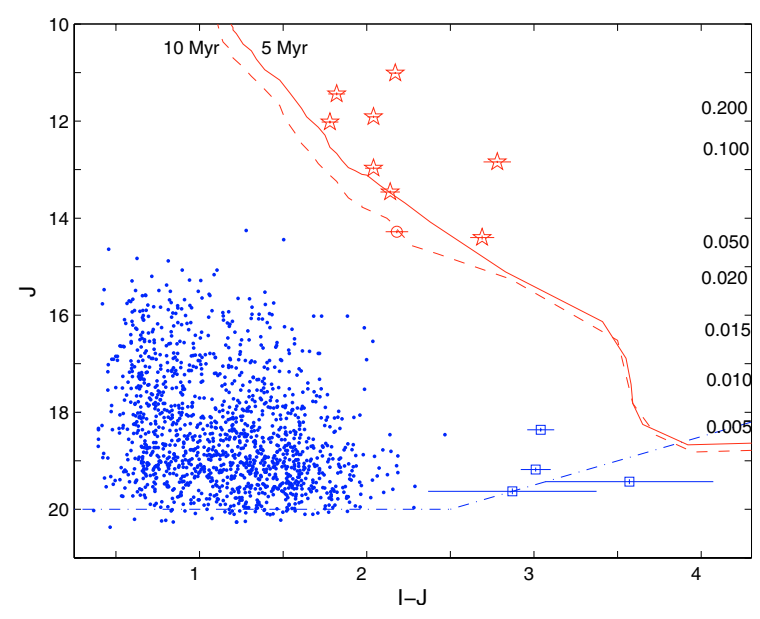

Fig. 3. $J, I-J$ colour-magnitude diagram. Previously known Upper Scorpius members are indicated with (red) stars. Probable nonmember UScoCTIO 121 is marked with a (red) circle. (Blue) squares denotes very cool objects found in our search and field stars are represented by (blue) dots. Solid and dashed (red) lines correspond to the 5 and 10 Myr theoretical isochrones from the Lyon group respectively (Baraffe et al. 1998; Chabrier et al. 2000). Limit magnitude is marked with a (blue) dot-dashed line. Theoretical masses $\left(M_{\odot}\right)$ for the $5-\mathrm{Myr}$ Lyon model, are shown to the right.

\subsection{Search for isolated low mass members}

We have also conducted a conventional search for free-floating substellar objects in our images building a $J, I-J$ colourmagnitude diagram for each field. In Fig. 3 we show the combination of all of them. Limiting magnitude is represented by a dot-dashed line. The 5 and $10 \mathrm{Myr}$ isochrones from evolutionary models of the Lyon group (Baraffe et al. 1998; Chabrier et al. 2000) are also plotted. We have converted their luminosity and $T_{\text {eff }}$ into $I-J$ colours and $J$ magnitudes following the prescriptions given before. From this diagram, we have selected the reddest object $(I-J \geq 2.5)$ identified in this survey. The photometry of these four ultracool objects are shown in Table 3. Since the optical and infrared images were not obtained simultaneously, the $I-J$ colour of these objects could be affected by intrinsic variability if they are really young objects.

From their $I-J$ and $J-K_{\mathrm{s}}$ colours, we determined that objects with identification numbers \#2 and \#3 correspond to dwarfs with M8 spectral type and that object \#1 has a spectral type later than M7-M 8 and earlier than L 6. The last object, identified as \#4, requires especial attention. According to the 
extremely red $I-J$ colour, the object is expected to have a spectral type at around L 5-L 7 with a very red $J-K_{\mathrm{S}}$ colour $(2.39 \pm 0.06)$. If the object is a field L 6 dwarf, it would be a brown dwarf with an estimated mass of $0.060-0.072 M_{\odot}$ (using Dusty00 models by Chabrier et al. 2000) located at a distance of around $135 \mathrm{pc}$. We cannot either rule out the possibility that object \#4 is a modestly extincted $\left(A_{\mathrm{V}}=2-3\right)$ isolated planetary-mass object in Upper Scorpius or an unresolved $\left(F W H M<1^{\prime \prime}\right)$ extremely red galaxy. We estimate from searches for extremely red objects (Thompson et al. 1999) that less than one such unresolved object might contaminate our survey.

According to their position in the $J, I-J$ colourmagnitude diagram, these objects show $J$ magnitude fainter and $I-J$ colours bluer than the $10 \mathrm{Myr}$ isochrone, which is the oldest age expected for the association, so they do not probably belong to the association. This conclusion is in agreement with the number of contaminants we estimate in our survey from the densities of field M, L and T dwarfs derived in Kirkpatrick et al. (1994), Cruz et al. (2003) and Burgasser et al. (2003), respectively. We expect that around 2 late $\mathrm{M}, 0.7 \mathrm{~L}$ and no $\mathrm{T}$ field dwarfs will be present in our search given the $I$-band limiting magnitude and the total area of the survey.

\section{Conclusions}

We have performed a search for substellar companions around eight very low-mass stars and brown dwarfs of the Upper Scorpius association. Using a PSF subtraction method, we have found three faint objects at 3-5 arcsec of the targets, but according to their $I-J$ colours, they seem to be background visual companions. In conclusion, we have not found any brown dwarfs more massive than $0.030 M_{\odot}$ at projected distances larger than $70 \mathrm{AU}$, and planets more massive than $0.007 M_{\odot}$ at projected distances larger than $600 \mathrm{AU}$. From the negative results of our search, we infer that the fraction of very-low mass stars and massive brown dwarfs with planets more massive than $\sim 0.007 M_{\odot}$ at physical distances larger than $600 \mathrm{AU}$ is lower than $20 \%$ (confidence level of $68.3 \%$ ). Using $J, I-J$ colour-magnitude diagrams and follow-up $K_{\mathrm{s}}$-band photometry, we have identified four ultracool candidates. According to their positions in the $J, I-J$ diagram, below the expected theoretical sequence of the cluster, three are probable late M/early L dwarfs in the field. The number of contaminants presented in our survey is consistent with our estimates. The faintest and reddest object $\left(J-K_{\mathrm{s}}=\right.$ $2.39 \pm 0.06)$ could be a field intermediate $\mathrm{L}$ dwarf with anomalously red $J-K_{\mathrm{s}}$ colour at a distance of $\sim 135 \mathrm{pc}$, an extremely red galaxy, or a reddened planetary-mass object in the Upper Scorpius association. A proper motion determination and follow-up spectroscopy are required to disentangle these possibilities.
Acknowledgements. We would like to thank to M. Rosa Zapatero Osorio for providing valuable software and Terry Mahoney for revising the English of the manuscript. A.M. and J.A.P. acknowledge support from grant AYA 2004-03136, financed by the Spanish Dirección General de Investigación.

\section{References}

Acosta-Pulido, J., Ballesteros, E., Barreto, M., et al. 2003, The Isaac Newton Group Newsletter, 7, 15

Ardila, D., Martín, E. L., \& Basri, G. 2000, AJ, 120, 479

Baraffe, I., Chabrier, G., Allard, F., \& Hauschildt, P. H. 1998, A\&A, 337,403

Burgasser, A. J., Kirkpatrick, J. D., Reid, I. N., et al. 2003, ApJ, 586, 512

Chabrier, G., Baraffe, I., Allard, F., \& Hauschildt, P. H. 2000, ApJ, 542,464

Chauvin, G., Lagrange, A.-M., Dumas, C., et al. 2004, A\&A, 425, 29

Cruz, K. L., Reid, N. I., Liebert, J., Kirkpatrick, J. D., \& Lowrance, P. J. 2003, AJ, 126, 2421

Cutri, R. M., Skrutskie, M. F., van Dyk, S., et al. 2003, VizieR Online Data Catalog: II/246. Originally published in: University of Massachusetts and Infrared Processing and Analysis Center (IPAC/California Institute of Technology)

de Zeeuw, P. T., Hoogerwerf, R., de Bruijne, J. H., J., Brown, A. G. A., \& Blaauw, A. 1999, AJ, 117, 354

Epchtein, N., et al. 1994, in Science with Astronomical Near Infrared Sky Suveys, ed. N. Epchtein, A. Omont, B. Burton \& P. Persi (Dordrecht: Kluwer), 3

Golimowski, D. A., Leggett, S. K., Marley, M. S., et al. 2004, AJ, 127, 3516

Gorlova, N. I., Meyer, M. R., Rieke, G. H., \& Liebert, J. 2003, ApJ, 593, 1074

Günther, E. W., \& Wuchterl, G. 2003, A\&A, 401, 677

Jayawardhana, R., Ardila, D., Stelzer, B., \& Haisch, K. E. 2003, AJ, 126,1515

Jayawardhana, R., Mohanty, S., \& Basri, G. 2002, ApJ, 578, L141

Kirkpatrick, J. D., McGraw, J., Hess, T. R., Liebert, J., \& McCarthy, D. W. 1994, ApJS, 94, 749

Leggett, S. K., Allard, F., Dahn, C., et al. 2000, ApJ, 535, 965

Leggett, S. K., Allard, F., Geballe, T. R., Hauschildt, P. H., \& Schweitzer, A. 2001, ApJ, 548, 908

Manchado, A., Barreto, M., Acosta-Pulido, J., et al. 2004, Proc. SPIE, ed. G. Hasinger, \& M. Turner, 5492, 1094

Mohanty, S., Basri, G., Jayawardhana, R., et al. 2004, ApJ, 609, 885

McCarthy, C., \& Zuckerman, B. 2004, 127, 2871

Muzerolle, J., Hillenbrand, L., Calvet, N., Briceño, C., \& Hartman, L. 2003, ApJ, 592, 266

Preibisch, T., Brown, A. G. A., Bridges, T., Günther, E., \& Zinnecker, H. 2002, AJ, 124, 404

Preibisch, T., Günther, E., \& Zinnecker, H. 2001, AJ, 121, 1040

Preibisch, T., \& Zinnecker, H. 1999, AJ, 117, 2318

Thompson, D., Beckwith, S. V. W., Fockenbrock, R., et al. 1999, ApJ, 523,100 
M. T. Costado et al.: Planetary-mass objects in Upper Scorpius, Online Material p 1

\section{Online Material}


M. T. Costado et al.: Planetary-mass objects in Upper Scorpius, Online Material p 2

\section{Appendix A: Tables}

Table A.1. Name, coordinates and $I J K_{\mathrm{s}}$ magnitudes of the targets.

\begin{tabular}{lccccc}
\hline \hline Name & $\alpha$ & $\delta$ & $I$ & $J$ & $K_{\mathrm{s}}$ \\
& $(\mathrm{J} 2000.0)$ & $(\mathrm{J} 2000.0)$ & $($ COUSINS $)$ & $(2 \mathrm{MASS})$ & $(2 \mathrm{MASS})$ \\
\hline UScoCTIO 121 & 155147.3 & -262347 & $16.49 \pm 0.07$ & $14.28 \pm 0.03$ & $13.32 \pm 0.04$ \\
UScoCTIO 128 & 155911.2 & -233759 & $17.12 \pm 0.07$ & $14.40 \pm 0.04$ & $13.21 \pm 0.04$ \\
UScoCTIO 112 & 160026.6 & -205632 & $15.63 \pm 0.06$ & $13.46 \pm 0.03$ & $12.51 \pm 0.02$ \\
UScoCTIO 56 & 160141.0 & -202208 & $13.98 \pm 0.04$ & $11.91 \pm 0.02$ & $10.86 \pm 0.02$ \\
UScoCTIO 100 & 160204.1 & -205042 & $15.65 \pm 0.09$ & $12.84 \pm 0.02$ & $11.83 \pm 0.03$ \\
USco J160622.8-201124 & 160622.8 & -201124 & $13.83 \pm 0.05$ & $12.02 \pm 0.03$ & $11.002 \pm 0.019$ \\
USco J160628.7-200357 & 160628.7 & -200357 & $13.29 \pm 0.05$ & $11.44 \pm 0.03$ & $10.464 \pm 0.019$ \\
USco J160629.0-205216 & 160629.0 & -205216 & $13.21 \pm 0.04$ & $11.01 \pm 0.02$ & $10.00 \pm 0.02$ \\
USco J160632.1-202053 & 160632.1 & -202053 & $15.04 \pm 0.05$ & $12.97 \pm 0.03$ & $11.94 \pm 0.02$ \\
\hline
\end{tabular}

${ }^{a}$ Probable non-member.

Table A.2. Name, main spectral properties and references of the targets.

\begin{tabular}{lccccc}
\hline \hline Name & $\begin{array}{c}E W(\mathrm{Li}) \\
(\AA)\end{array}$ & $\begin{array}{c}E W(\mathrm{H} \alpha) \\
(\AA)\end{array}$ & $\begin{array}{c}\text { Spectral } \\
\text { type }\end{array}$ & Remarks $^{a}$ & References $^{b}$ \\
\hline UScoCTIO 121 & $\leq 0.3$ & -10.0 & M6 & Nm & $1,6,9$ \\
UScoCTIO 128 & 0.5 & -130.5 & M7 & $L$, lowg, Em & $1,4,5,6,7,9$ \\
UScoCTIO 112 & 0.6 & -16.1 & M5.5 & $E m$ & $1,4,7,9$ \\
UScoCTIO 56 & - & -9.1 & M5 & - & 1,2 \\
UScoCTIO 100 & 0.6 & -16.1 & M7 & $E m$ & $1,4,5,6,7,8,9$ \\
USco J160622.8-201124 & 0.53 & -6.0 & M5 & - & 2,3 \\
USco J160628.7-200357 & 0.58 & -30.0 & M5 & - & 2,3 \\
USco J160629.0-205216 & 0.68 & -6.2 & M5 & - & 2,3 \\
USco J160632.1-202053 & 0.69 & -5.1 & M5 & - & 2,3 \\
\hline
\end{tabular}

${ }^{a}$ Nm.: Probable non-member; $L$ : Excess in the $L^{\prime}$ band; lowg: Low surface gravity; $E m$ : Presence of lines in emission.

${ }^{b}$ References: (1) Ardila et al. (2000); (2) Preibisch et al. (2001); (3) Preibisch et al. (2002); (4) Jayawardhana et al. (2002); (5) Gorlova et al. (2003); (6) Muzerolle et al. (2003); (7) Jayawardhana et al. (2003); (8) Günther \& Wuchterl (2003); (9) Mohanty et al. (2004). 


\section{Appendix B: Observations and data analysis}

\section{B.1. Optical observations}

We obtained Cousins I-band images using the Optical Imager Galileo (OIG) instrument, mounted on a Nasmyth platform of the Telescopio Nazionale Galileo at the Roque de los Muchachos Observatory, La Palma, on 2002 June 28-30. This optical camera has two $2048 \times 4096$ pixel EEV 42-80 CCD detectors, with a plate scale of $0.072 \mathrm{arcsec} / \mathrm{pixel}$, and covering a total area of $4.9 \times 4.9 \mathrm{arcmin}^{2}$ in each exposure. There is a physical separation between both detectors of $\sim 2.8$ arcsec. Spatial binning of $2 \times 2$ pixels was used during our observations (hence, giving a real pixel size of $0.144 \mathrm{arcsec}$ ). Nights were photometric and average seeing ranged from 0.6 to 0.9 arcsec. Raw data were bias-subtracted using the overscan region and flat-field corrected. Flat-field images for each night were obtained combining sky flat-field images taken at dusk. For the reduction we used routines from the imred package within the IRAF $^{1}$ environment.

\section{B.2. Infrared observations}

We obtained $J$-band images using the INGRID instrument, mounted on the Cassegrain focus of the $4.2 \mathrm{~m}$ William Herschel Telescope at the Roque de los Muchachos Observatory, La Palma, on 2002 March 26. INGRID is a near-infrared camera which consists of a $1024 \times 1024$ pixel $^{2}$ Hawai'i detector, providing a plate-scale of 0.238 arcsec/pixel, and covering a total area of $4.06 \times 4.06 \mathrm{arcmin}^{2}$ in each exposure. The night was photometric and the average seeing was of 1.1 arcsec. Observations were performed using a dithering pattern of nine positions. For each field, the total exposure time was $9 \times 4 \times$ $30 \mathrm{~s}=1080 \mathrm{~s}$.

$K_{\mathrm{s}}$-band images for all the fields except for USco J160632.1-202053 were obtained with the CAIN-2 instrument, mounted on the $1.55 \mathrm{~m}$ Telescopio Carlos Sánchez at the Teide Observatory on 2002 June 7-9. The CAIN-2 near-infrared camera has a $256 \times 256$ Nicmos-3 detector, and in its wideoptic mode provides a plate scale of $1.00 \mathrm{arcsec} / \mathrm{pixel}$ and a total area of $4.27 \times 4.27 \mathrm{arcmin}^{2}$. The nights were not photometric (uniform layer of thin cirrus), that slightly affects the limiting magnitude of our observations, and the average seeing was 1.5 arcsec. We performed the observations using a dithering pattern of ten positions, with 6-12 exposures of $20-10 \mathrm{~s}$ in each position, giving a total exposure time of $1800 \mathrm{~s}$. The $K_{\mathrm{s}}$-band image of the USco J160632.1-202053 and UScoCTIO 128 fields were obtained with the LIRIS instrument mounted on the Cassegrain focus of the $4.2 \mathrm{~m}$ William Herschel Telescope on 2004 June 8 and 10, as part of the Guaranteed Time programme. Thus, two different $K_{\mathrm{s}}$-band images for UScoCTIO 128 were obtained. The LIRIS instrument is a new near-infrared camera and spectrograph, built at the Instituto de Astrofísica de Canarias (Acosta-Pulido et al. 2003;

${ }^{1}$ IRAF is distributed by National Optical Astronomy Observatories, which is operated by the Association of Universities for Research in Astronomy, Inc., under contract with the National Science Foundation.
Manchado et al. 2004). It uses a $1024 \times 1024$ Hawai'i detector, providing a plate scale of $0.250 \mathrm{arcsec} /$ pixel and a total area of $4.27 \times 4.27 \mathrm{arcmin}^{2}$. The nights were photometric and the average seeing was in the range of 1.2-1.5 arcsec. A five-point dithering pattern was used, obtaining 15 frames of $8 \mathrm{~s}$ exposure time at each point, which means an exposure time per cycle of $600 \mathrm{~s}$. This cycle was repeated six times giving a total exposure time of $3600 \mathrm{~s}$.

All infrared data were processed in the same way. Raw data were sky-subtracted and flat-field corrected. We generated sky frames for each field and filter combination by median-filtering the dithered scientific images to eliminate the point sources. Flat-field images were derived combining sky flat-field images taken at dawn and dusk for the INGRID and CAIN-2 data. Dome flat-field images were obtained for the LIRIS data. Images were then aligned and combined. For the reduction we used several routines within the IRAF environment.

\section{B.3. Photometric analysis}

For all the images obtained in different filters we carried out the same photometric analysis. We used routines from the daophot package within IRAF. Point sources were selected using the daofind task, so extended sources were mostly avoided. We performed aperture and psf photometry of the selected objects using the phot routine. To transform instrumental into real magnitudes we used the photometry of objects in common with our images and several existing catalogues. For the calibration of the $J$ and $K_{\mathrm{s}}$-band, we used the photometry from the 2MASS catalogue (Cutri et al. 2003) and for the $I$-band data we used the photometry from the DENIS catalogue (DENIS Consortium 2003, Epchtein et al. 1994). We have transformed the $I$-band data from DENIS into the Cousins system, using $I_{\mathrm{C}}-i_{\text {DENIS }}=0.030 \pm 0.04$, obtained by comparison of the photometry of $\mathrm{M}$ and $\mathrm{L}$ dwarfs from Legget et al. (2000, 2001) and DENIS. We performed an independent calibration for each field and filter. Estimates for the completeness magnitudes of the $I-, J$ - and $K_{\mathrm{s}}$-band images are $21.6 \pm 0.5$, $19.2 \pm 0.5,17.1 \pm 0.5$, respectively. Limiting magnitudes of the $I-, J$ - and $K_{\mathrm{s}}$-band images are $22.5 \pm 0.5,20.0 \pm 0.5,17.8 \pm$ 0.5 , respectively. For the $K_{\mathrm{s}}$ images obtained with LIRIS, the completeness and limiting magnitudes are $18.5 \pm 0.5$ and 19.5 \pm 0.5 , respectively. We adopted as completeness magnitude the value at which the histogram of detected objects per magnitude reaches a maximum, and as limiting magnitude the value at which it drops below half of the maximum (these are roughly equivalent to a $10 \sigma$ and $3 \sigma$ detection limits, respectively).

\section{Appendix C: Detectability of nearby companions (statistical analysis)}

If we consider that the number of objects with possible companions is well represented by a Poisson distribution, the probability of having no companions is given by:

$$
P[x \equiv 0]=\left[\mathrm{e}^{-\lambda} \frac{\lambda^{x}}{x !}\right]_{x \equiv 0}=\mathrm{e}^{-\lambda}
$$


where $\lambda$ is the Poisson parameter, $\lambda=n p$, being $n$ the number of events ( 8 observed Upper Scorpius members) and $p$, the real fraction of companions. We can determine, for a confidence level (C.L.), where $P=1-$ C.L., that the upper limit to the real fraction of substellar companions is $p=-\ln (P) / n$.

In order to know the real fraction of substellar companions at physical (not projected) distances, it is necessary to correct this result by the probability, $p^{\prime}$, that the orbital position of the companion is at a detectable projected distance. This correction factor assumes dramatic importance when the angle of inclination, $i$, between the plane of the orbit and the plane of the sky is close to $\pi / 2$ and the semimajor axis is small. The projection of a circular orbit on the plane of the sky is an ellipse with eccentricity $\sin i$ :

$\rho(r, \theta, i)=\frac{r}{\left(1+\tan ^{2} i \sin ^{2} \theta\right)^{1 / 2}}$, where $r$ is the orbital radius and $\theta \in[0,2 \pi)$. Assuming that all the angular inclinations of the orbit are equally probable and that orbits are circular, the probability $p^{\prime}$ is given by:

$p^{\prime}\left(i_{0}\right)=\frac{2}{\pi} i_{0}+\frac{4}{\pi^{2}} \int_{i_{0}}^{\frac{\pi}{2}} \mathrm{~d} i \arcsin \left(\frac{\tan i_{0}}{\tan i}\right)$,

where $i_{0}(r)=\arccos \left(r_{0} / r\right)$ and $r_{0}$ is the projected distance from which a particular companion can be detected. In this case,

$\lambda(r)=\sum_{1}^{\mathrm{n}} p_{j}^{\prime}(r) p$

where $p_{j}^{\prime}(r)$ is the probability that a companion orbiting at a distance $r$ is at a detectable separation from the object $j$. In a similar way than before, we can determine, for a confidence level and a physical separation $r$, the upper limit to the real fraction of substellar companions. 